\title{
Manifestações Contratransferenciais no Processo Terapêutico de uma Paciente com Personalidade Borderline
}

\author{
Rochele Luciane Möller' \\ Fernanda Barcellos Serralta ${ }^{1}$ \\ Aline Alvares Bittencourt \\ Silvia Pereira da Cruz Benetti ${ }^{1}$ \\ ${ }^{1}$ UNISINOS, São Leopoldo, RS
}

\begin{abstract}
Resumo
Pacientes com transtorno de personalidade borderline (TPB) são tipicamente instáveis e intensos em seus afetos e comportamentos, facilmente despertando reações emocionais negativas em seus terapeutas. Desse modo, manejar a contratransferência é fundamental para a psicoterapia com esses pacientes. Este estudo de caso sistemático visa descrever as manifestações contratransferenciais no processo terapêutico do primeiro ano de uma terapia psicanalítica de uma paciente com TPB, ampliando o conhecimento empírico sobre como a contratransferência interage com as variáveis do paciente. As sessões $(n=59)$ foram codificadas por duplas de juízes independentes treinados com o Psychotherapy Process Q-Set (PQS), um q-sort de 100 itens que fornece descrição das variáveis do paciente, do terapeuta e da interação terapêutica que caracterizam o tratamento. Experts em psicoterapia psicanalítica selecionaram seis itens do PQS como indicadores de reações contratransferenciais e 12, como indicadores de estados mentais e comportamentos característicos de pacientes com TPB. Esses itens foram examinados em relação ao tempo. Os achados revelam a predominância de atitudes terapêuticas compatíveis com uma contratransferência positiva. As implicações clínicas dos achados são discutidas.

Palavras-chave: processo psicoterápico, transtorno de personalidade borderline, contratransferência
\end{abstract}

\section{Countertransferential Manifestations in the Therapeutic Process of a Patient with Borderline Personality}

\begin{abstract}
Patients with borderline personality disorder (BPD) are typically unstable and intense in their feelings and behaviors, easily arousing negative emotional reactions in their therapists. Therefore, managing countertransference is essential for psychotherapy with these patients. The present systematic case study aims to describe countertransferential manifestations in the therapeutic process of the first year of a psychoanalytical therapy with a BPD patient, broadening the empirical knowledge on how countertransference interacts with the patient's variables. The sessions $(n=59)$ were codified by pairs of independent judges trained with the Psychotherapy Process Q-Set (PQS), a q-sort with 100 items that give a description of the patient, the therapist and of the interaction variables that typify the treatment. Experts in psychoanalytical psychotherapy selected six PQS items as indicative of countertransferential reactions and 12, as indicators of mental states and behaviors characteristic of patients with BPD. These items were examined in relation to time. Findings reveal predominance of therapeutic attitudes compatible with positive countertransference.
\end{abstract}

Keywords: Psychotherapeutic process; borderline personality disorder; countertransference

\section{Manifestaciones Contratransferenciales en el Proceso Terapéutico de una Paciente con Personalidad Borderline}

\section{Resumen}

Pacientes con trastorno de personalidad borderline (TPB) son típicamente inestables e intensos en los afectos y comportamientos, y despiertan fácilmente reacciones emocionales negativas en los terapeutas. Por lo tanto es fundamental manejar la contratransferencia en la psicoterapia con estos pacientes. Este estudio de caso sistemático pretende describir las manifestaciones contratransferenciales en el proceso terapéutico del primer año de una terapia psicoanalítica de un paciente con TPB, ampliando el conocimiento empírico sobre, cómo la contratransferencia interactúa con las variables del paciente. Las sesiones $(n=59)$ fueron codificadas por duplas de jueces independientes, entrenados con el Psycoterapy Process Q-Set (PQS), un q-sort de 100 ítems que proporcionan descripción de las variables del paciente, del terapeuta y de la interacción terapéutica que caracterizan el tratamiento. Expertos en psicoterapia psicoanalítica seleccionaron 6 ítems del PQS como indicadores de reacciones contratransferenciales y 12, como indicadores de estados mentales y comportamientos característicos de pacientes con TPB. Estos ítems fueron examinados con relación al tiempo y los resultados revelan predominio de actitudes terapéuticas compatibles con una contratransferencia positiva. Se discuten las implicaciones clínicas de los resultados.

Palabras-clave: proceso psicoterápico; trastorno de personalidad borderline; contratransferencia 


\section{Introdução}

O Transtorno de Personalidade Borderline (TPB) tem sido considerado um dos transtornos psiquiátricos mais difíceis de tratar (Bateman, \& Fonagy, 2004; Sulzer, 2015). Ainda que diversas modalidades de psicoterapia sejam efetivas para o tratamento do TPB (Stoffers et al., 2012), pacientes com esse diagnóstico costumam ser vistos como um desafio ao psicoterapeuta (McMain, Boritz, \& Leybman, 2015). Os transtornos de personalidade implicam em padrões disfuncionais de percepção, relação e pensamento sobre si mesmo e outros, expressos em contextos sociais e interpessoais (DSM-5, APA, 2014). Os padrões relacionais mal adaptativos dos pacientes com TPB influenciam o relacionamento terapêutico, resultando, entre outros fatores, em dificuldades no estabelecimento e manutenção da aliança terapêutica, na adesão ao tratamento e na evocação de intensos sentimentos de contratransferência. Portanto, o modo como o terapeuta responde às dificuldades interpessoais e à desregulação emocional desses pacientes é crucial para determinar o sucesso ou o insucesso do tratamento (McMain et al., 2015).

Pessoas com TPB tipicamente apresentam um padrão difuso e pervasivo de instabilidade nos relacionamentos, na autoimagem e nos afetos, além de marcada impulsividade, evidenciados em pelo pelos cinco dos seguintes indicadores: esforços para evitar o abandono real ou imaginário, sentimentos crônicos de vazio, alternância entre extremos de idealização e desvalorização nos relacionamentos interpessoais, alterações no senso de identidade, comportamento impulsivo, comportamento suicida ou de automutilação, instabilidade do humor, dificuldade para controlar a raiva, ideação paranoide reativa e transitória ou sintomas dissociativos severos. O transtorno é mais frequentemente diagnosticado em mulheres e que possui alta prevalência, entre $1,6 \%$ e $5,9 \%$, na população geral. Entre pacientes de consultórios e ambulatórios de saúde mental, a prevalência é de aproximadamente $10 \%$. Entre pacientes internados, a prevalência atinge 20\% (DSM-5, APA, 2014).

$\mathrm{Na}$ psicanálise, a designação borderline possui significado menos preciso que na psiquiatria, remetendo a uma forma particular de estruturação psíquica e modalidades características de estabelecer relações de objeto (Pasini, \& Dametto, 2010). Conforme modelo proposto por Otto Kernberg, a personalidade borderline é uma organização estável que tem como características centrais: difusão do self (baixo controle de impulsos), defesas primitivas centradas na cisão (negação, projeção e introjeção), testagem da realidade intacta (pensamento de processo primário) e relacionamentos objetais internalizados patológicos (controle do objeto e limitada empatia) (Kernberg, Selzer, Koenigsberg, Carr, \& Appelbaum, 1991; Lenzenweger, Clarkin, Levy, \& Kernberg, 2012). De modo geral, entende-se que tais características têm raízes numa perturbação precoce do desenvolvimento que impede a constância objetal, já que as suas relações com as figuras parentais foram afetivamente indiferentes ou com uma intimidade aparente, o que predispõe à dependência simbiótica do objeto materno e ausência/demissão do objeto paterno (Maranga, 2002).

Ao não conseguirem estabelecer as fronteiras do eu e dos objetos, pacientes borderlines lutam para evitar a perda ou o abandono, não tolerando a solidão, e vivenciando angústia intensa, de aniquilamento e morte (Kernberg et al., 1991; Maranga, 2002; Pasini, \& Dametto, 2010). Esses pacientes apresentam constante necessidade de utilizar o objeto como uma segunda pele mental, sendo que a perda do objeto é vivida como a perda de si próprio (Dias, 2004). Ao experienciar frustrações ocasionadas pelo ambiente, sentem raiva desmedida e desespero, não aceitando esses sentimentos como parte de si, e sim percebidos como vindos de fora (Yeomans, Levy, \& Caligor, 2013).

Esses padrões disfuncionais com frequência se manifestam no relacionamento terapêutico, imprimindo no setting terapêutico uma constante pressão. Violações de limites, falta de comprometimento com o tratamento e agressividade dirigida ao terapeuta são comuns, podendo despertar intensas reações de contratransferência negativa (Bateman, \& Fonagy, 2004; Clarkin, Yeomans, \& Kernberg, 1999; Gunderson 2009; Linehan, 1993; Schestatsky, 2005; Wildgoose, Waller, Clarke, \& Reid, 2000). Por esse motivo, considera-se que o exame cuidadoso das respostas emocionais de terapeutas em relação à personalidade desses pacientes é de grande valia para orientar o manejo terapêutico (Bateman, \& Fonagy, 2004; Colli, \& Ferri, 2015). Visando o controle das reações emocionais de paciente e terapeuta e a construção de um relacionamento terapêutico positivo, entre as estratégias comuns nas psicoterapias baseadas em evidências para pacientes com TPB estão o foco na consciência das emoções, a responsividade, a maior estruturação do tratamento, a supervisão e envolvimento da equipe, e a exploração das rupturas da aliança (McMain et al., 2015). 
A contratransferência é um constructo central na clínica psicanalítica. Conforme revisão de Molina e Fabrian (2014), na história do pensamento psicanalítico, o construto foi concebido de forma diferente por diferentes grupos de autores. Por exemplo, na perspectiva clássica de Freud e dos autores da primeira geração, constitui um entrave ao processo, uma reação inconsciente (resíduo não analisado) do analista; para Ferenczi e autores da escola britânica, compreende a totalidade das reações emocionais do terapeuta (incluindo os sentimentos despertados pelo paciente, que servem para a sua compreensão); para autores franceses e autores latino-americanos da atualidade, a contratransferência serve para compreender a situação analítica e, junto com a transferência, constitui o campo analítico. No âmbito da investigação empírica, o estudo das reações contratransferenciais ganhou força somente durante as últimas décadas, e ainda necessita aprimoramento quanto aos seus métodos de avaliação (Gelso, \& Hayes, 2007; Hayes, 2004; Hayes, Gelso, \& Hummel, 2011; Rosenberger, \& Hayes, 2002).

Com a intenção de descrever os principais estudos que avaliaram a contratransferência na psicoterapia de adultos, nas diferentes abordagens psicoterápicas, a revisão sistemática de Machado et al. (2014) nas bases de dados Embase, PubMed, PsycINFO e Web of Knowledge, identificou que a maioria das pesquisas foram conduzidas nos Estados Unidos e tratavam de psicoterapia psicodinâmica conduzidas por estudantes ou psicoterapeutas em treinamento. Foram encontradas poucas investigações que incluíam pacientes com transtorno de personalidade e o desenvolvimento da contratransferência durante o curso da terapia.

Nota-se ainda que a maior parte dos estudos de contratransferência utiliza delineamentos transversais e sugere que os aspectos do funcionamento do paciente suscitam diferentes reações emocionais no terapeuta (Betan, Heim, Conklin, \& Westen, 2005; Betan, \& Westen, 2009; Brody, \& Farber, 1996; Hayes, Gelso, \& Hummel, 2011; Meehan, Levy, \& Clarkin, 2012; Rossberg, Karterud, Pedersen, \& Friis, 2008/2010). Geralmente, raiva e pouca empatia são as respostas emocionais mais frequentemente suscitadas por pacientes com personalidade borderline (Brody, \& Farber, 1996; Yeomans, Levy, \& Caligor, 2013).

Com vistas a contribuir para a investigação empírica da contratransferência na psicoterapia psicanalítica, este estudo tem o objetivo de descrever as manifestações da contratransferência no processo do primeiro ano de uma terapia psicanalítica de uma paciente com personalidade borderline e examinar possíveis associações entre tais manifestações e estados mentais negativos e comportamentos da paciente que tipicamente estão presentes em indivíduos com TPB, tais como depressão, ansiedade, raiva, culpa, desconfiança e controle. Considerando a literatura, a expectativa é de encontrar associações que indiquem que estados mentais da paciente (em especial os negativos) estão associados a reações sugestivas de contratransferência negativa da terapeuta. Com este estudo intensivo e sistemático de caso, pretende-se contribuir para o desenvolvimento de conhecimentos baseados em dados empíricos sobre como a contratransferência se manifesta e interage com as variáveis do paciente, buscando auxiliar na compreensão do processo terapêutico de pacientes supostamente mais difíceis e possibilitar reflexão sobre formas de manejo clínico destes casos.

\section{Método}

\section{Delineamento}

Este é um estudo de caso sistemático (ECS) que busca compreender o processo terapêutico, com uso de medidas repetidas e metodologia mista (Dattilio, Edwards, \& Fishman, 2010).

\section{Caso em Estudo}

O caso é de uma psicoterapia de orientação psicanalítica. A paciente Mariana, 22 anos, buscou tratamento em uma clínica psiquiátrica por se cortar em momentos de raiva e ter crises intensas de choro. Possuía história de vários tratamentos interrompidos. Após uma primeira avaliação, a paciente foi encaminhada para atendimento psiquiátrico e psicoterápico. O psiquiatra responsável diagnosticou-a com transtorno de personalidade borderline (DSM-IV-TR, APA, 2002). Esse diagnóstico foi corroborado pela avaliação clínica da psicoterapeuta e pela Shedler-Westen Assessment Procedure-200 (Shedler, \& Westen, 2007). Nessas avaliações, destacam-se os traços proeminentes de ansiedades paranoides e de desregulação emocional. Sentia-se deprimida e ansiosa, tendia a ver os outros como pessoas más que a rejeitam e respondia com raiva inapropriada e/ou se autoagredindo. A terapeuta possuía nove anos de experiência clínica e treinamento formal em psicoterapia psicanalítica em uma instituição local, cujo modelo de formação é baseado no tripé psicanalítico: seminários teóricos, supervisões e tratamento pessoal. A psicoterapia iniciou com frequência de duas vezes na semana, mas após os 
primeiros meses, por solicitação da paciente, passou a ocorrer semanalmente.

\section{Instrumento}

\section{Psychotherapy Process Q-Set (PQS)}

Para avaliação geral do processo terapêutico, utilizou-se o Psychotherapy Process Q-Set (PQS). O PQS foi desenvolvido por Enrico Jones (2000) e é uma medida do tipo q-sort, composta por um total de 100 itens que fornecem descrições dos comportamentos, atitudes, estados mentais e outras características do terapeuta $(n$ $=41)$, do paciente $(n=40)$ e a relação terapêutica $(n=$ 19), podendo ser utilizado para descrever o processo terapêutico de diferentes psicoterapias. A elaboração da versão em português do PQS foi desenvolvida por Serralta, Nunes e Eizirik (2007) e apresentou níveis de fidedignidade interavaliadores semelhantes aos obtidos com a versão original.

O PQS é acompanhado de um manual de aplicação e a sua utilização requer treinamento prévio e tipicamente é feita por duplas de avaliadores independentes. Após estudar a sessão terapêutica gravada em áudio e/ou vídeo, os juízes ordenam os 100 itens em uma escala ipsativa (forçada em acordo com a curva normal) de nove pontos, indo do extremo não característico ou negativamente saliente (categoria 1) ao extremo característico ou saliente (categoria 9).

O PQS não é um instrumento construído para avaliação específica da contratransferência. Não obstante, por constituir uma medida global do processo, alguns itens desse instrumento contemplam aspectos do constructo contratransferência. Para identificar os indicadores de manifestações da contratransferência, solicitou-se que psicólogos experts em psicoterapia psicanalítica $(n=6)$, com experiência clínica mínima de 6 anos, indicassem os itens relacionados aos aspectos das reações contratransferenciais. Os psicólogos apontaram que os itens mais expressivos da contratransferência são os que avaliam sensibilidade e empatia do terapeuta aos sentimentos do paciente (Q6; $n=6$ ), distância e indiferença versus responsividade e envolvimento (Q9; $n=5)$, autorrevelação (Q21; $n=5)$, interferência dos conflitos emocionais na relação terapêutica $(\mathrm{Q} 24 ; n=6)$, flexibilidade para melhorar a relação terapêutica $(\mathrm{Q} 47 ; n=4)$ e atitudes condescendentes ou protetoras para com o paciente $(\mathrm{Q} 51 ; n=5)$.

Para selecionar os itens que são característicos de estados mentais e comportamentos de pacientes com TPB e que presumidamente podem evocar reações contratransferenciais no terapeuta, o mesmo procedimento de avaliação por experts foi realizado. Foram identificados 12 itens: paciente dirige sentimentos agressivos dirigidos ao terapeuta (Q1;n=6), está ansioso (Q7; $n=5)$, é provocador $(\mathrm{Q} 20 ; n=6)$, experimenta afetos dolorosos (Q26;n=6), culpa os outros pelas suas dificuldades (Q34; $n=5)$, se sente cauteloso ou desconfiado ( $\mathrm{Q} 44 ; n=5)$, tem sentimentos ambivalentes com o terapeuta (Q49; $n=5)$, se sente inferior versus superior (Q59; $n=6)$, é exigente (Q83;n= 5), expressa sentimentos negativos (Q84; $n=6)$, é controlador $(\mathrm{Q} 87 ; n=6)$ e se sente triste ou deprimido (Q94; $n=6)$.

\section{Procedimentos de Coleta, Análise e Éticos}

A psicoterapia está atualmente em andamento, mas para fins deste estudo, foram consideradas somente as sessões do primeiro ano do tratamento, totalizando 59 sessões.

Todas estas sessões $(n=59)$ foram gravadas em vídeo e aleatoriamente avaliadas por duplas de avaliadores independentes previamente treinados para a codificação com o PQS.

Em todas as sessões, correlações intraclasse foram computadas para verificar a concordância entre os avaliadores, tendo como critério correlações iguais ou superiores a 0,7 . Nas sessões em que este critério não foi atingido, um terceiro avaliador foi utilizado. Nas análises subsequentes, foi utilizada a média das avaliações dos dois juízes concordantes.

A descrição do processo terapêutico do primeiro ano da psicoterapia foi realizada por meio do ordenamento dos 10 itens do PQS mais e menos salientes. Para descrever as manifestações da contratransferência do primeiro ano da psicoterapia, foi feita uma descrição com os itens do PQS que melhor indicavam as reações contratransferenciais, conforme avaliação de experts (Q6, Q9, Q21, Q24, Q47 e Q51).

Procedimentos de correlação (Pearson) foram utilizados para verificar a relação entre a contratransferência e variáveis da paciente. Estas últimas também foram identificadas pelos experts a partir dos itens do PQS (Q1, Q7, Q20, Q26, Q34, Q44, Q49, Q59, Q83, Q84, Q87 e Q94). Uma vez que o PQS possui escala do tipo $Q$ em que os extremos indicam sentidos opostos, nos resultados, itens com sentido reverso foram identificados com a letra $\mathrm{R}$ após o número do item. $\mathrm{O}$ nível de significância adotado foi de 5\%. Todos os procedimentos estatísticos foram realizados no programa SPSS, versão 21.0. 
$\mathrm{Na}$ condução deste estudo foram respeitados os princípios éticos da pesquisa envolvendo os seres humanos, na qual paciente e a terapeuta assinaram o Termo de Consentimento Livre e Esclarecido (TCLE). O protocolo do estudo foi aprovado pelo Comitê de Ética em Pesquisa na universidade de origem.

\section{Resultados}

Para descrever as características do processo do primeiro ano de tratamento de Mariana medidas pelo PQS, foram consideradas as médias dos 10 itens mais e menos salientes, incluindo variáveis da paciente, da terapeuta e da interação terapêutica. A Tabela 1 apresenta a média e o desvio padrão dos itens encontrados.

Possíveis manifestações contratransferenciais foram verificadas por meio dos itens do PQS (Q6, Q9,
Q21, Q24, Q47 e Q51). Utilizou-se o recurso gráfico para identificar a variação destes itens ao longo do processo no primeiro ano de tratamento.

Conforme pode ser visualizado na figura 1 , ao longo do processo, a terapeuta mostrou-se sensível e empática aos sentimentos da paciente (Q6) em todas as sessões (exceto na sessão 52), e responsiva e efetivamente envolvida (Q9R). Quanto ao fato da terapeuta revelar informações sobre si (Q21), houve variações: em algumas sessões esta atitude foi característica e em outras, não característica. Este item foi classificado como "razoavelmente característico ou saliente", nas sessões 16, 20, 35, 51, 52, 54 e 56. A maior classificação no extremo não característico foi obtida na sessão 11 e 23.

No que se refere à interferência dos conflitos emocionais da própria terapeuta na relação terapêutica (Q24), a sessão inicial e as sessões 6, 18, 19, 22,

Tabela 1

Itens do PQS Mais e Menos Característicos do Processo Terapêutico Durante o $1^{\circ}$ Ano

\begin{tabular}{|c|c|c|c|}
\hline item & Descrição & $\mathrm{m}$ & $\mathrm{Dp}$ \\
\hline & Itens mais característicos & & \\
\hline 31 & A terapeuta solicita mais informação ou elaboração. & 8,11 & 0,64 \\
\hline 69 & A situação de vida atual ou recente da paciente é enfatizada na discussão. & 7,84 & 1,34 \\
\hline 6 & A terapeuta é sensível aos sentimentos da paciente, afinada com a paciente; empática. & 7,82 & 0,74 \\
\hline 46 & A terapeuta se comunica com a paciente com um estilo claro e coerente. & 7,52 & 0,78 \\
\hline 86 & A terapeuta é segura ou autoconfiante. & 7,50 & 0,85 \\
\hline 88 & A paciente traz temas significativos. & 7,42 & 1,62 \\
\hline 18 & A terapeuta transmite um sentido de aceitação não crítica. & 7,19 & 0,92 \\
\hline 54 & A paciente é clara em sua expressão. & 7,16 & 1,11 \\
\hline 28 & A terapeuta percebe acuradamente o processo terapêutico. & 7,11 & 0,89 \\
\hline 63 & Os relacionamentos interpessoais da paciente são um tema importante. & 6,97 & 1,66 \\
\hline \multicolumn{4}{|c|}{ Itens menos característicos } \\
\hline 9 & A terapeuta é distante, indiferente (versus responsiva e efetivamente envolvida). & 2,07 & 0,87 \\
\hline 77 & A terapeuta não tem tato. & 2,10 & 0,90 \\
\hline 53 & A paciente está preocupada com o que a terapeuta pensa dela. & 2,14 & 0,91 \\
\hline 25 & A paciente tem dificuldade em começar a sessão. & 2,38 & 1,50 \\
\hline 87 & A paciente é controladora. & 2,67 & 1,17 \\
\hline 61 & A paciente se sente tímida ou envergonhada (versus à vontade e segura). & 2,70 & 1,00 \\
\hline 78 & A paciente busca aprovação, afeição, ou simpatia da terapeuta. & 2,73 & 1,04 \\
\hline 5 & A paciente tem dificuldade para compreender os comentários da terapeuta. & 2,91 & 1,92 \\
\hline 44 & A paciente se sente cautelosa ou desconfiada. & 2,91 & 1,31 \\
\hline 15 & A paciente não inicia assuntos; é passiva. & 3,00 & 2,13 \\
\hline
\end{tabular}

Nota PQS $=$ Psychotherapy Process Q-Set, $m=$ média; $d p=$ desvio padrão

Psico-USF, Bragança Paulista, v. 23, n. 4, p. 705-717, out./ dez: 2018 


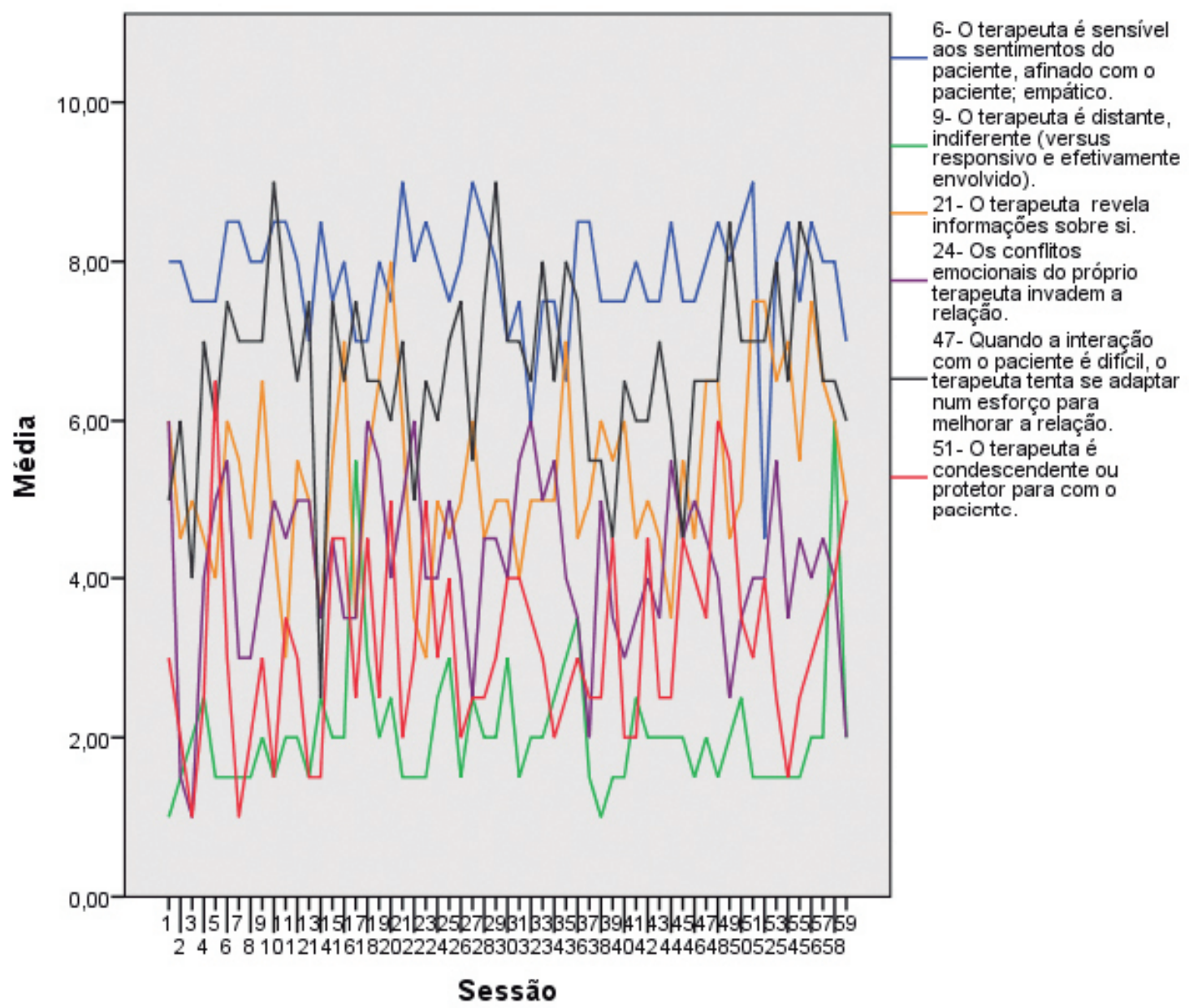

Figura 1. Variação da contratransferência (itens Q6, Q9, Q21, Q24, Q47 e Q51 do PQS) no processo terapêutico.

32, 34, 44 e 53 foram classificadas entre o "neutro" e "um pouco característico ou saliente". Porém, considerando-se o processo como um todo, este item se manteve no extremo não característico, indicando que a ausência desta interferência é digna de nota. Foi constatado ainda que quando a interação com a paciente era difícil, a terapeuta era flexível e tentava se adaptar num esforço para melhorar a relação terapêutica (Q47). Essa atitude foi extremamente característica em um número muito significativo de sessões, atingindo pontuação máxima (escore 9) nas sessões 10 e 29. De modo geral, a figura indica que a terapeuta não foi condescendente ou protetora para com a paciente $(\mathrm{Q} 51 \mathrm{R})$, pois este item teve pouca variação e foi basicamente classificado como extremo não característico.

A seguir, foi verificada a associação entre os itens do PQS que refletem manifestações contratransferenciais e os itens que avaliam atitudes, comportamentos e estados mentais da paciente.

Verificou-se que os itens que avaliam interferência dos conflitos emocionais da própria terapeuta na relação terapêutica (Q24) bem como que indicam indiferença e falta de responsividade (Q9) não apresentaram correlação significativa com nenhuma das variáveis da paciente.

A menor sensibilidade e empatia da terapeuta (Q6) apresentou associação significativa com maior ansiedade e tensão da paciente (Q7; r $=-0,32, p=0,004)$, sua tendência a culpar outros, ou forças externas, pelas suas dificuldades (Q34; $r=-0,33, p=0,019)$ e mais controle desta da interação com a terapeuta (Q87; $r=-, 0,27, p$ $=0,038$ ).

A autorrevelação da terapeuta (Q21) apresentou associação com sentimentos de inadequação ou inferioridade da paciente $(\mathrm{Q} 59, r=0,27, p=0,041)$ e com 
Tabela 2

Correlações entre Indicadores de Contratransferência da Terapeuta e Variáveis da Paciente

\begin{tabular}{ccccccc}
\hline & \multicolumn{7}{c}{ Terapeuta } \\
\cline { 2 - 6 } & Q6 & Q9 & Q21 & Q24 & Q47 & Q51 \\
\hline Paciente & - & - & - & - & $0,57 * *$ & - \\
Q1 & $-0,32 *$ & - & - & - & - & - \\
Q7 & - & - & - & - & $0,53 * *$ & - \\
Q20 & - & - & - & - & - & - \\
Q26 & $-0,33 *$ & - & - & - & - & - \\
Q34 & - & - & - & - & $-34^{* *}$ & $-0,31^{*}$ \\
Q44 & - & - & - & - & - & - \\
Q49 & - & - & $0,27 *$ & - & - & - \\
Q59 & - & - & - & - & - & - \\
Q83 & $-0,27 *$ & - & $-0,38^{* *}$ & - & - & - \\
Q84 & - & - & - & - & - & - \\
Q87 & - & - & - & - & - \\
Q94 & - & - & - & - & - & - \\
\hline
\end{tabular}

Nota. Q6 = sensibilidade e empatia; Q9 = distância e indiferença; Q21 = autorrevelação; Q24 = conflitos emocionais; Q47 = adaptação; Q51= condescendência e proteção; Q1 = sentimentos agressivos dirigidos ao terapeuta; Q7 = ansiedade; Q20 = provoca e desafia; Q26 = experimenta afetos dolorosos ; Q34 = culpa os outros; Q44 = cautelosa ou desconfiada; Q49 = ambivalente com terapeuta; Q59 = se sente inferior versus superior; Q83 = exigente Q84= expressa sentimentos negativos; Q87 = controladora; Q94 = se sente triste ou deprimida; * $p \leq 0,05$; ** $p \leq$ 0,01. - = correlação não significativa.

menor expressão de sentimentos de raiva (Q84, $r=$ $0,31, p=0,015)$.

A necessidade da terapeuta em adaptar-se à paciente (Q47) apresentou correlação positiva significativa com a verbalização de sentimentos negativos dirigidos a terapeuta (Q1; $r=0,55, p=0,011)$, com atitudes de provocação e desafios aos limites da relação terapêutica (Q20; $r=0,53, p=0,000)$, com a desconfiança (Q44; $r=0,34, p=0,009)$ e com a tendência a fazer demandas e exigências em excesso (Q83; $r=0,31$, $p=0,015)$.

Atitudes de maior condescendência e proteção por parte da terapeuta (Q51) estiveram associadas a maior desconfiança da paciente na terapeuta (Q44R; $r$ $=-0,31, p=0,017)$ e menor ambivalência (Q49; $r=$ $0,28, p=0,032)$

\section{Discussão}

Mariana iniciou seu tratamento devido a queixas características de TPB. A despeito das suas dificuldades interpessoais e do seu histórico de psicoterapias interrompidas, durante o primeiro ano do tratamento mostrou, de modo geral, atitudes de cooperação para com sua terapeuta, parecendo disposta a receber ajuda e suporte psicológico oferecido. Ainda que dificuldades de manejo clínico de pacientes com personalidade borderline sejam comuns devido às suas tentativas de perverter os objetivos de tratamento e de formar vínculos não terapêuticos e de intimidade com o terapeuta (Tanesi, 2007; Zimerman, 2004), a descrição do primeiro ano da psicoterapia de Mariana, com base nas avaliações obtidas com o PQS, sugere que a sensibilidade, envolvimento e empatia da terapeuta, bem como sua flexibilidade em adaptar-se ao funcionamento da paciente na sessão tenham atuado como promotoras de um trabalho colaborativo da dupla.

De modo geral, a descrição do processo terapêutico obtida com o PQS sugere uma interação terapêutica fluída e benigna, não marcada por intensas manifestações contratransferenciais. A análise visual da variação dos itens indicadores de manifestações contratransferenciais, realizada com auxílio do recurso gráfico de dispersão, confirma o achado de uma prevalência de atitudes positivas da terapeuta para com a paciente, 
sendo a empatia e responsividade, as características mais marcantes.

Nota-se que, em consonância com a descrição geral do processo que indica uma terapeuta predominantemente responsiva, alguns indicadores mais diretos de reações de contratransferência negativa, como, por exemplo, a intrusão de conflitos emocionais da terapeuta (Q24) e atitudes de indiferença e falta de responsividade (Q9) não se associaram as variáveis da paciente. Neste sentido, o padrão geral de respostas emocionais da terapeuta de Mariana está em consonância com os estudos de Thylstrup e Hesse (2008) que encontraram, entre profissionais da saúde, uma menor intensidade das reações contratransferenciais com pacientes com personalidade borderline do que o sugerido na literatura. Segundo os autores, as reações mais frequentes foram o desejo de ajudar e ser prestativo, o que parece também ter ocorrido, em grande medida, com a terapeuta de Mariana.

Contudo, o exame das correlações significativas encontradas ajuda a lançar luz sobre aspectos não tão fluídos da interação não captados pela simples descrição das características mais prevalentes no tratamento. Ressalta-se que, conforme esperado, diversos indicadores de reações contratransferenciais, mostraram associação com atitudes e estados mentais da paciente. Constatou-se, por exemplo, que a empatia da terapeuta é menor quando mariana está mais ansiosa, culpa forças externas por suas dificuldades (ao invés de aceitar a sua participação nos problemas) e exerce maior controle na interação com a terapeuta. Esses achados são coerentes com a previsão derivada das pesquisas que indicam que pacientes com menor nível de funcionamento psicológico podem produzir mais respostas negativas no terapeuta e menor tendência à empatia tendência de empatia (Brody, \& Farber, 1996). Essa é também uma constatação frequente na clínica com estes pacientes (Yeomans, Levy, \& Caligor, 2013).

Conforme indicado na figura 1 , em diversos momentos a terapeuta fez esforços para se adaptar ao funcionamento da paciente, buscando melhorar a relação. E, mais, foi constatado que tais esforços estão associados a maior intensidade de diversas atitudes típicas de pacientes com TPB, tais como verbalização de sentimentos agressivos dirigidos à terapeuta, excesso de demandas, comportamentos provocadores e sentimentos paranoides ou de desconfiança com a terapeuta. Estas atitudes da terapeuta indicam que, de alguma forma, estava consciente das pressões exercidas pela paciente e ajustava-se a elas sem reagir, confrontar ou fazer interpretações de conteúdo. Isso é coerente com a assertiva de que com funcionamento mais regressivo exigem mais adaptações à técnica e cuidado na manutenção da aliança terapêutica (Bateman, \& Fonagy, 2004; Yeomans, Levy, \& Caligor, 2013). Assim, entende-se que a terapeuta foi mais flexível diante de indicadores de transferência negativa. Sabe-se que tal atitude, nas psicoterapias psicodinâmicas é consistentemente associada a melhores resultados do que a adesão rígida aos princípios da técnica (Owen, \& Hilsenroth, 2014).

A literatura aponta também que com pacientes TPB, as estratégias técnicas eficazes incluem a responsividade e autenticidade do terapeuta (McMain et al., 2015). Uma manifestação clara de flexibilidade na técnica adotada é a autorrevelação, característica da atitude da terapeuta em várias sessões (vide figura 1). A tendência da terapeuta de revelar sentimentos e outros aspectos de si mesma mostrou associação com mais sentimentos de inferioridade e menos de raiva ou agressividade heterodirigida. Ainda que psicoterapeutas de orientação psicanalítica tradicionalmente apontem a revelação de sentimentos e dados pessoais do terapeuta na sessão como atitude que prejudica a expressão da transferência, estudos recentes mostram que a autorrevelação pode ser benéfica para pacientes mais regressivos, na medida em que o terapeuta se apresenta como pessoa "real", o que pode contribuir para a diminuição de sentimentos de isolamento, por exemplo (Howe, 2011).

Em consonância com as atuais concepções de contratransferência, entende-se que a fim de não obstruir a empatia e a compreensão, o terapeuta precisa reconhecer a presença de intrusões contratransferenciais (Samberg, \& Marcus, 2007; Wolff, \& Falcke, 2011). Se adequadamente contida, a ansiedade que o terapeuta desenvolve na contratransferência pode servir como uma possibilidade de acesso e aproximação, ou seja, transformada em capacidade empática que juntamente com a capacidade interpretativa, contém e transforma a angústia do paciente (Romaro, 2002; Rosa, \& Santos, 2011).

A crença de que o paciente com TPB pode beneficiar-se com o tratamento psicoterápico, expressa uma atitude sensível por parte do terapeuta (Lequesne, \& Hersh, 2004). Isso provavelmente ocorreu no tratamento de Mariana que parece ter encontrado, após múltiplas tentativas mal sucedidas, uma terapeuta experiente e continente. Dentro desta perspectiva, concordamos com Romaro (2002, p. 7) quando afirma que, "os objetivos dos serviços e dos processos terapêuticos 
precisariam ser repensados, ressaltando-se os aspectos contratransferenciais, assim, talvez, o paciente borderline não fosse à priori considerável intratável, mas tratável dentro de um enquadramento mais específico e estruturado, com objetivos delimitados".

É bem possível pressupor que a experiência clínica, o treinamento e o tratamento pessoal da terapeuta de Mariana possam ter colaborado na capacidade em lidar terapeuticamente com as suas próprias reações emocionais, utilizando-as como um instrumento valioso para compreender a paciente. Na relação terapêutica de pacientes com personalidade borderline, a maior experiência clínica e o treinamento profissional do terapeuta estão associados a mais reações positivas ao paciente, pois ajudam o terapeuta a compreender melhor as suas reações e o instrumentalizam para a tomada de decisões de tratamento (Liebman, \& Burnette, 2013). O uso da contratransferência, sugerido pelas auto revelações da terapeuta de Mariana e os esforços adaptativos diante das manifestações de transferência negativa possivelmente indicam uma atenção da terapeuta aos afetos da paciente e aos seus próprios, atitude que tem sido relacionada à promoção da aliança terapêutica nas terapias com pacientes com TPB (McMain et al., 2015).

Quando se trata de pacientes com TPB, terapeutas podem cair em rótulos de diagnóstico estereotipados, que transportam conotações de periculosidade e intratabilidade (Schulze, 2007), gerando desesperança e enfraquecimento da aliança terapêutica (Aviram, Brodsky, \& Stanley, 2006; Markham, \& Trower, 2003). Por outro lado, estudos mostram que a expectativa de contratransferência se relaciona ao transtorno do paciente e também à formação teórica do terapeuta (Gordon et al., 2016). Neste sentido, efetuar um diagnóstico adequado e conhecer os desafios que determinado transtorno apresenta, aliados à sensibilidade clínica pode ser fundamental para que eventuais reações emocionais não sejam simplesmente atuadas e sim manejadas terapeuticamente, ou seja, auxiliem a conduzir o tratamento em consonância com as características do paciente.

Este é um estudo de caso naturalístico e que não investigou a relação entre processos e resultados. Entende-se que as generalizações são teóricas e que existe a necessidade de investigações semelhantes com outros casos para ampliar a validade externa do estudo. A associação entre indicadores de contratransferência e variáveis da paciente foi feita por meio de procedimentos de correlação, que não permitem presumir relações de causa-efeito. Considerando que a contratransferência é um constructo difícil de ser operacionalizado e mensurado empiricamente, a utilização de um instrumento específico para a sua avaliação, talvez pudesse proporcionar a captação de aspectos mais específicos e sutis das emoções da terapeuta. Ressalta-se, entretanto, que apesar do PQS não ter sido construído para a investigação das reações contratransferenciais, o instrumento foi capaz de fornecer informações relevantes sobre o comportamento, atitudes e intervenções da terapeuta que conforme psicoterapeutas de orientação psicanalítica são indicadores destas reações.

\section{Considerações Finais}

O sofrimento psíquico de pacientes com personalidade borderline não se restringe somente aos indivíduos que possuem o transtorno de personalidade, mas também afeta os vínculos de quem convive com ele. Como o padrão predominantemente hostil destes pacientes se estende em suas demais relações, repetindo-se inclusive no setting terapêutico, cabe ao terapeuta viabilizar um melhor manejo terapêutico, cujas adaptações em relação à técnica precisam ser adequadas de acordo com as suas peculiaridades.

No caso analisado, verifica-se a predominância de atitudes terapêuticas fundamentadas numa contratransferência sutil e positiva. Isso pressupõe o uso da própria mente e mundo interno para compreender o outro e ajustar as intervenções às suas capacidades e necessidades. Neste sentido, o estudo está em consonância com a ideia de que pacientes com TPB podem ser mal compreendidos por terapeutas com pouco treinamento ou que não realizam tratamento pessoal, o que pode contribuir para o rótulo amplamente difundido de que estes são pacientes difíceis e provocam intensas reações emocionais em seus terapeutas. Não se trata de minimizar as dificuldades técnicas que podem se apresentar na psicoterapia psicanalítica de pacientes com TPB, mas sim de questionar a intratabilidade nestes casos e ressaltar o papel protetivo da experiência clínica, do treinamento e da psicoterapia ou análise pessoal. A consciência dos sentimentos contratransferenciais pode servir como um valioso instrumento de trabalho para melhor compreender a ausência ou presença da empatia com estes pacientes.

No entanto, é preciso ter ressalvas quanto ao prognóstico de um processo terapêutico fluído e de um trabalho colaborativo em andamento. Lembramos que este estudo se propôs a investigar somente o primeiro ano de tratamento e não focaliza o processo de mudança, sintomatológica e/ou estrutural. Além 
disto, o uso da média dos itens do q sort utilizado para avaliação do processo fornece tão somente uma visão global do que caracterizou o primeiro ano de tratamento. Isso pode obscurecer variações importantes nas sessões. Neste sentido, as associações encontradas entre diversos indicadores de contratransferência e sentimentos e atitudes da paciente alertam para situações de conflito e dificuldades na interação que podem ocorrer. $\mathrm{O}$ abandono do processo terapêutico é demasiadamente frequente, o que corrobora para um entusiasmo não excessivo por parte do terapeuta e uma expectativa cautelosa em relação ao trabalho clínico. Essa atitude pode prevenir rupturas na aliança não identificadas (e, portanto, não trabalhadas) pelo terapeuta.

O presente artigo foi inovador em termos da abordagem metodológica prevista para explorar a contratransferência. A investigação empírica de constructos psicanalíticos é complexa, mas possível, desde que se considere também suas limitações. Não foram investigadas as reações emocionais inconscientes da terapeuta e sim atitudes e comportamentos que, conforme juízes experts, podem indicar reações contratransferenciais. Buscou-se, portanto, examinar derivações observáveis do fenômeno. Neste sentido, o PQS mostrou-se útil e promissor. Entretanto, em pesquisas futuras, recomenda-se a inclusão de instrumentos já validados para o estudo da contratransferência, assim como análises de microprocessos.

Os estudos dedicados à investigação das reações contratransferenciais de pacientes com personalidade borderline no processo terapêutico não são conclusivos, o que justifica a proposição de novas pesquisas de processo-resultados que focalizem a relação entre paciente e terapeuta, a fim de aprimorar a compreensão das particularidades e as intervenções clínicas com estes pacientes, além de testar diferentes caminhos instrumentais e metodológicos para a avaliação de constructos psicanalíticos.

\section{Referências}

American Psychiatric Association - APA. (2014). Manual diagnóstico e estatístico de transtornos mentais: DSM-5. (5a ed.). Porto Alegre: Artmed.

Aviram, R. B., Brodsky, B. S., \& Stanley, B. (2006). Borderline personality disorder, stigma and treatment implications. Harvard Review of Psychiatry, 14(5), 249-256. doi: 10.1080/10673220600975121
Bateman, A. W., \& Fonagy, P. (2004). Psychotherapy for borderline personality disorder: Mentalization-based treatment. New York: Oxford University Press.

Betan, E. J., \& Westen, D. (2009). Countertransference and personality pathology: Development and clinical application of the countertransference questionnaire. Em Levy, R. A., \& Ablon, J. S. Handbook of evidence-based psychodynamic psychotherapy: Bridging the gap between science and practice. New York: Human Press, 179-198. doi: 10.1007/978-1-59745-444-5_8

Betan, E. J., Heim, A. K., Conklin, C. Z., \& Westen, D. (2005). Countertransference phenomena and personality pathology in clinical practice: An empirical investigation. The American Journal of Psychiatry, 162(5), 890-898. doi: 10.1176/appi.ajp.162.5.890

Brody, E., \& Farber, B. A. (1996). The effects of therapist experience and patient diagnosis on countertransference. Psychotherapy: Theory, Research, Practice, Training, 33(3), 372-380. doi: 10.1037/0033-3204.33.3.372

Clarkin, J. F., Yeomans, F. E., \& Kernberg, O. F. (1999). Psychotherapy for borderline personality. New York: Wiley.

Colli, A., \& Ferri, M. (2015). Patient personality and therapist countertransference. Current opinion in psychiatry, 28(1), 46-56. doi: 10.1097/ YCO.0000000000000119

Colli, A., Tanzilli, A., Dimaggio, G., \& Lingiardi, V. (2014). Patient personality and therapist response: An empirical investigation. Am J Psychiatry, 171(1), 102-108. doi: 10.1176/appi.ajp.2013.13020224

Dattilio, F. M., Edwards, D. J. A., \& Fishman, D. B. (2010). Case studies within a mixed methods paradigm: Toward a resolution of the alienation between researcher and practitioner in psychotherapy research. Psychotherapy: Theory, Research, Practice, Training, 47(4), 427-441. doi: 10.1037/a0021181

Dias, C. A. (2004). Costurando as linhas da psicopatologia borderland (estados-limite). Lisboa: Climepsi.

Gelso, C. J., \& Hayes, J. A. (2007). Countertransference and the therapist's inner experience: Perils and possibilities. Mahwah, N.J: Lawrence Erlbaum Associates.

Gordon, R. M., Gazzillo, F., Blake, A., Bornstein, R. F., Etzi, J., Lingiardi, V., ... \& Tasso, A. F. (2016). The relationship between theoretical orientation and countertransference expectations: Implications for 
ethical dilemmas and risk management. Clinical psychology \& psychotherapy, 23(3), 236-245.

Gunderson, J. G. (2009). Borderline personality disorder: Ontogeny of a diagnosis. The American Journal of Psychiatry, 166(5), 530-539. doi: 10.1176/appi. ajp.2009.08121825

Hayes, J. A. (2004). The inner world of the psychotherapist: A program of research on countertransference. Psychotherapy Research, 14(1), 21-36. doi: $10.1093 / \mathrm{ptr} / \mathrm{kph} 002$

Hayes, J. A., Gelso, C. J., \& Hummel, A. M. (2011). Managing countertransference. Psychotherapy, 48(1), 88-97. doi: 10.1037/a0022182

Howe, E. (2011). Should Psychiatrists Self Disclose? Innovations in Clinical Neuroscience, 8(12), 14-17. Retrieved from https://www.ncbi.nlm.nih.gov/ pmc/articles/PMC3257982/

Jones, E. E. (2000). Therapeutic action: A guide to psychoanalytic therapy. New Jersey: Aronson.

Kernberg, O., Selzer, M., Koenigsberg, H., Carr, A., \& Appelbaum, A. (1991). Psicoterapia psicodinâmica de pacientes borderline. Porto Alegre: Artes Médicas.

Lenzenweger, M. F., Clarkin, J. F., Levy, K. N., \& Kernberg, O. F. (2012). Predicting domains and rates of change in borderline personality disorder. Personality Disorders: Theory, Research and Treatment, 3(2), 185-195. doi: 10.1037/a0025872

Lequesne, E., \& Hersh, R. (2004). Disclosure of a diagnosis of borderline personality disorder. Journal of Psychiatric Practice, 10(3), 170-176. doi: 10.1097/00131746-200405000-00005

Liebman, R., \& Burnette, M. (2013). It's not you, it's me: An examination of clinician - and client-level influences on countertransference toward borderline personality disorder. American Journal of Orthopsychiatry, 83(1), 15-125. doi: 10.1111/ajop.12002

Linehan, M. (1993). Cognitive-behavioral treatment of borderline personality disorder. New York: Guilford Press.

Machado, D., Coelho, F., Giacomelli, A., Donassolo, M., Abitante, M., Dall'Agnol, T., \& Eizirik, C. (2014). Systematic review of studies about countertransference in adult psychotherapy. Trends in Psychiatry and Psychotherapy, 36(4), 173-185. doi: 10.1590/2237-6089-2014-1004
Maranga, A. R. (2002). Organizações borderline: Aspectos psicodinâmicos. Análise Psicológica, 20(2), Lisboa. doi: 10.14417/ap.307

Markham, D., \& Trower, P. (2003). The effects of the psychiatric label 'borderline personality disorder' on nursing staff's perceptions and causal attributions for challenging behaviours. British Journal of Clinical Psychology, 42(3), 243-256. doi: 10.1348/01446650360703366

McMain, S. F., Boritz, T. Z., \& Leybman, M. J. (2015). Common strategies for cultivating a positive therapy relationship in the treatment of borderline personality disorder. Journal of Psychotherapy Integration, 25(1), 20.

Meehan, K. B., Levy, K. N., \& Clarkin, J. F. (2012). Construct validity of a measure of affective communication in psychotherapy. Psychoanalytic Psychology, 29(2), 145-165. doi: 10.1037/a0027450

Owen, J., \& Hilsenroth, M. J. (2014). Treatment adherence: The importance of therapist flexibility in relation to therapy outcomes. Journal of Counseling Psychology, 61(2), 280-288. doi: 10.1037/a0035753

Pasini, T. F., \& Dametto, J. (2010). Abordagem psicodinâmica do paciente borderline. Perspectiva, Erechim, 34(128), 133-149. Recuperado de http://www.uricer.edu.br/site/pdfs/perspectiva/128_143.pdf

Romaro, R. A. (2002). O sentimento de exclusão social em personalidade borderline e o manejo da contratransferência. Revista Mudanças, 10(1), 62-71. Recuperado de http://www.ritaromaro.com.br/wp-content/uploads/2014/06/ artigo_o_sentimento_de_exclusao_social_em_ personalidade_borderline.pdf

Rosa, B. P., \& Santos, M. A. (2011). Comorbidade entre bulimia e transtorno de personalidade borderline: Implicações para o tratamento. Revista latinoamericana de psicopatologia fundamental, 14(2), 268-282. doi: 10.1590/S1415-47142011000200005

Rosenberger, E. W., \& Hayes, J. A. (2002). Therapist as subject: A review of the empirical countertransference literature. Journal of Counseling and Development, 80(3), 264-270. doi: 10.1002/j.1556-6678.2002. tb00190.x

Rossberg, J. I., Karterud, S., Pedersen, G., \& Friis, S. (2008). Specific personality traits evoke different countertransference reactions: An empirical study. 
Journal of Nervous and Mental Disease, 196(9), 702708. doi: 10.1097/NMD.0b013e318186de80

Rossberg, J. I., Karterud, S., Pedersen, G., \& Friis, S. (2010). Psychiatric symptoms and countertransference feelings: An empirical investigation. Psychiatry Research, 178(1), 191-195. doi: 10.1016/j. psychres.2009.09.019

Samberg, E., \& Marcus, E. (2007). Processo, resistência e interpretação. Em Person, E. S., Cooper, A. M., \& Gabbard, G. O. (org.). Compêndio de Psicanálise. Porto Alegre: Artmed, 237-247.

Schestatsky, S. S. (2005). Abordagem psicodinâmica do paciente borderline. Em Eizirik, C., Aguiar, R., \& Schestatsky, R. S. Psicoterapia de orientação analítica: Fundamentos teóricos e clínicos. Porto Alegre: Artmed.

Schulze, B. (2007). Stigma and mental health professionals: A review of the evidence on an intricate relationship. International Review of Psychiatry, 19(2), 137-155. doi: 10.1080/09540260701278929

Serralta, F., Nunes, M., \& Eizirik, C. (2007). Elaboração da versão em português do Psychotherapy Process Q-Set. Revista de Psiquiatra do Rio Grande do Sul, 29(1), 44-55. doi: 10.1590/S0101-81082007000100011.

Shedler, J., \& Westen, D. (2007). The Shedler-Westen Assessment Procedure (SWAP): Making personality diagnosis clinically meaningful. Journal of Personality Assessment, 89(1), 41-55. doi: 10.1080/00223890701357092

Stoffers, J. M., Völlm, B. A., Rücker, G., Timmer, A., Huband, N., \& Lieb, K. (2012). Psychological therapies for people with borderline personality disorder. Cochrane Database of Systematic Revienus, 8, CD005652.
Sulzer, S. H. (2015). Does “difficult patient" status contribute to de facto demedicalization? The case of borderline personality disorder. Social Science \& Medicine, 142, 82-89. doi: 10.1016/j. socscimed.2015.08.008

Tanesi, P., Yazigi, L., Fiore, M., \& Pitta, J. (2007). Adesão ao tratamento clínico no transtorno de personalidade borderline. Estudos de Psicologia, 12(1), 71-78. doi: 10.1590/S1413-294X2007000100009

Thylstrup, B., \& Hesse, M. (2008). Substance abusers' personality disorders and staff members' emotional reactions. BMC Psychiatry, 8(21), 21-27. doi: 10.1186/1471-244X-8-21

Wildgoose, A., Waller, G., Clarke, S., \& Reid, A. (2000). Psychiatric symptomatology in borderline and other personality disorders: Dissociation and fragmentation as mediators. Journal of Nervous and Mental Disease, 188(11), 757-763. doi: 10.1097/00005053-200011000-00006

Wollf, C., \& Falcke, D. (2011). A contratransferência na clínica psicanalítica contemporânea. Análise Psicológica, 29(2), 201-214. Recuperado de <http:// www. scielo.mec.pt/scielo.php? script $=$ sci_ arttext\&pid $=$ S0870-82312011000200002\&lng $=p t$ $\& \mathrm{nrm}=$ iso

Yeomans, F., Levy, K., \& Caligor, E. (2013). Transference-focused psychotherapy. Psychotherapy, 50(3), 449-453. doi: 10.1037/a0033417

Zimerman, D. E. (2004). Manual de técnica psicanalítica: Uma re-visão. Porto Alegre: Artmed.

Recebido em: 03-09-2017 Reformulado em: 13-03-2018

Aprovado em: 03-05-2018 
Sobre as autoras:

Rochele Luciane Möller é psicóloga, especialista em Psicoterapia de Orientação Psicanalítica (IPSI-Novo Hamburgo, RS) e mestre em Psicologia Clínica (UNISINOS-São Leopoldo, RS). Exerce atividades como psicoterapeuta em clínica privada. Endereço: Rua Heller, n. 510, apto. 404, CEP: 93510-330, Centro, Novo Hamburgo - RS.

E-mail: rochelemoller@gmail.com

ORCID: https://orcid.org/0000-0003-2531-7066

Fernanda Barcellos Serralta é psicóloga, especialista em psicoterapia psicanalítica (IEPP-Porto Alegre, RS), doutora em ciências médicas: psiquiatria (UFRGS), professora e pesquisadora no Programa de Pós-Graduação em Psicologia da UNISINOS - São Leopoldo, RS. Seu foco de estudo são os processos e os resultados de psicoterapias psicodinâmicas. É também a atual presidente do Capítulo Latino Americano da Society for Psychotherapy Research (SPR).

E-mail:fserralta@unisinos.br

ORCID: https://orcid.org/0000-0003-4602-6495

Aline Alvares Bittencourt é psicóloga, especialista em Psicoterapia de Orientação Psicanalítica (ESIPP - Porto Alegre, RS), mestre em Psicologia Clínica e doutoranda no Programa de Pós-Graduação em Psicologia da UNISINOS - São Leopoldo, RS. Exerce atividades como psicoterapeuta em clínica privada e é docente no ESIPP.

E-mail:alinealvares@hotmail.com

ORCID: https://orcid.org/0000-0001-7287-5832

Silvia Pereira da Cruz Benetti é psicóloga, especialista em psicoterapia psicanalítica (NEP-Porto Alegre, RS), doutora em Estudos da Criança e da Família (Syracuse University). Atualmente é pesquisadora colaboradora em diversos grupos de pesquisa, com foco nos estudos sobre a adolescência as psicoterapias psicodinâmicas.

E-mail: spcbenetti@gmail.com

ORCID: https://orcid.org/0000-0001-8557-2216

Contato com as autoras:

Rochele Luciane Möller

Rua Heller, n. 510, apto. 404, Centro

Novo Hamburgo-RS, Brasil

CEP: 93510-330 